\title{
Application of Vapor Phase Soldering (VPS) for Joints Reliability Improvement
}

\author{
Agata Skwarek, Beata Synkiewicz, Krzysztof Witek, and Sebastian Wronski
}

\begin{abstract}
The reliability of an electronic system may depend on solder joint work. Various methods are used at present to improve joint reliability One possibility is vapor phase soldering (VPS). The biggest advantages of VPS are high efficiency and uniform heating characteristics. It is also possible to ensure good control of the soldering temperature, an oxygen free soldering atmosphere, and, especially a higher rate of heat transfer. Joints made with VPS can be more uniform with fewer defects than in joints made with reflow soldering. However, the choice of solder alloy used for VPS may be a key factor in the quality of the junction.
\end{abstract}

Index Terms-Solder joints, vapor phase soldering, ceramic elements.

\section{INTRODUCTION}

The ever growing miniaturization of electronic components and electronic circuits has posed questions about their mounting technologies and protection. The reliability of an electronic system may depend on solder joint work. That is why it is important to select the correct methods and optimize the parameters of the soldering process to ensure the best quality of joint. As reported earlier, the reliability of solder joints has even been regarded as the most critical issue in SMT because of the large strains which develop at the interconnection between the components and the substrate material during operation [1]. These strains may cause severe cracking and premature failure in solder joints [2]. It is desirable to achieve a good metallurgical bond in the formation of the thin intermetallic compound layer which arises from the reaction between the solder and the substrate.

However, excessive intermetallic compound growth may have deleterious effects [3], [4]. Both stress concentration and the intermetallics formed at the solder/conductor interface are other factors in the failure of solder joints [2]. The solder joint defects that have been observed and analyzed can be divided into two types, external and structural. The external ones are those that can be detected with the naked eye or with optical instruments. These include short circuits between fields and leads (solder bridges), missing or improper solder joint appearances, the transfers of

Manuscript received April 16, 2013; revised June 28, 2013. The study was partially supported by the European Union within the European Regional Development Fund, through the Innovative Economy grant no POIG.01.01.02-00-108/09 (MIME).

Agata Skwarek, Beata Synkiewicz, Krzysztof Witek are with Institute of Electron Technology, Zablocie 39, 30-424 Krakow, Poland (e-mail: askwarek@ite.waw.pl)

Sebastian Wronski is AGH- University of Science and Technology, Al. Mickiewicza 30, 30-059 Krakow, Poland (e-mail: sebastian.wronski@gmail.com) electronic components for the pads, incorrect marking elements, tombstoning - the effect of the vertical lift component on the solder etc.

Structural defects inside solder joints are much harder to find and remove. Their characteristics are practically undetectable by conventional tests and their emergence in the production in the long term maybe due to climatic factors (an increase in temperature or humidity) or mechanical ones (vibration, shocks). Often, they lead to sudden and irreversible damage to the electronics, which in extreme cases can have disastrous consequences. Among these effects, tin pest, tin whiskers [5], [6], cold joints, dendrites and solder voids pose the greatest threats to circuit reliability.

Improving the soldering process should lead to the better solder joint quality. Any special requirements of a samples destination should be taken into consideration at the planning stage. Where the choice of the proper soldering method is essential. Vapor phase soldering is a viable candidate for ensuring better results.

\section{A. Vapor Phase Soldering}

Vapor phase soldering (also known as condense soldering) was discovered and patented in the early 70's. Its popularity has varied during the last 40 years. Nowadays the biggest advantages of VPS are seen as a stable temperature during the process, an oxygen free atmosphere for soldering and a higher rate of heat transfer.

In VPS technology, the increase of thermal energy (required to create solder joints) comes from the condensation of saturated vapor on the surface of the device. The vapor phase is generated by heating up a dense medium fluid (in this case Galden) to a stable boiling temperature. The heat transfer is dependent on the temperature difference between the vapor and the device and stops immediately when both reach the boiling point of the medium. This completely eliminates the risk of the overheating. These facts give VPS an undeniable advantage over any reflow process.

VPS is performed in special furnaces of different sizes and of a fixed construction. In these furnaces, the heating of the samples which have to be soldered is followed by using the heat energy of the vapor. The energy is released by the medium during the transition from the gaseous to the liquid state. The vapor is produced by heating a liquid (medium) of high density which has a constant boiling point. The soldering samples are immersed in saturated vapor which is condensed immediately into relatively cold elements. The saturated vapor temperature corresponds to the boiling point of the liquid used as the medium and remains constant during the change of phase. When the elements reach the temperature of the vapor the condensation process stops. The maximum temperature of the sample cannot exceed the 
boiling point of the medium. After moving the samples from the heating zone, the vapor of the medium is evaporated [7]. A scheme of the VPS process is presented in Fig. 1.

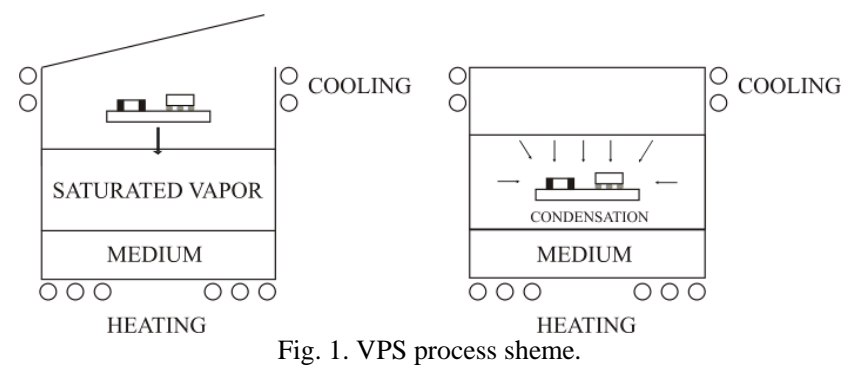

Additionally, the furnace can be equipped with a vacuum option which can be very useful for the reduction of voids as a result of flux residues being taken out.

\section{EXPERIMENTAL}

To asses the quality of joints made with the vapor phase soldering technique, test samples were prepared using PCB technology. Glass-epoxy laminates with a $\mathrm{Cu}$ foil $17 \mu \mathrm{m}$ thick were coated with a UV-sensitive photo-resist. The pads were tailored to 1206 SMD resistors. To cure the photoresist, the samples were exposed to ultra-violet light. Then, the samples were exposed to ferric chloride. The areas of $\mathrm{Cu}$ which were uncovered by photo-resist were etched with ferric chloride. At the next stage of the experiment, the $\mathrm{Cu}$ pads were covered with a protective coating of electroless $\mathrm{Ni} / \mathrm{Au}$ or layers of galvanic $\mathrm{Sn}$. Then the solder alloys Sn96.5Ag3Cu0.5 and Sn99.3Cu0.7Ni were applied using the screenprinting method. 1206 SMD resistors were placed with a Quadra pick-and place machine. Both vapor phase (with and without vacuum option) and reflow soldering methods were used for the soldering process.

The quality of the solder joints (from the reflow process and the VPS technique with a vacuum device) was assessed for mechanical strength, void content and their appearance under the microscope.

For visual analysis samples were observed in a Hirox KH7700 microscope, which allows 30-3500 times magnification and the possibility of multifocus synthesis for higher resolution and high focal depth.

The mechanical strength of solder joints was tested by a shear force analysis after exposure to thermal shocks, the force being applied to elements occurring in a direction parallel to the surface of the substrate.

For the investigation of solder voids, three dimensional $\mathrm{X}$-Ray images of samples were taken by the Phoenix Nanotom S computed tomography system with a 180 $\mathrm{kV} / 15 \mathrm{~W}$ nanofocus.

\section{RESULTS}

\section{A. Visual Analysis}

The shape of the joint can be a fundamental factor in joint quality. Solder joint geometry has major influence for example on the thermal fatigue lifetime of the joint. It was proved that the lack of a proper shape of a bump solder joint in BGA led to the electrical failure of the joint after exposure to thermal cycling. The joint shape influences joint cracking and as a result an increase in electrical resistance. Experimental data suggested that shape is the dominant factor affecting the onset of cracking. The reason for cracking is growing internal strain [8]. The proper shape also ensures the optimum consumption of the materials used for the soldering process.

As the observation shows, the quality of joints soldered by the traditional reflow process is lower. The joint surfaces are more irregular and the shape suggests holes, cracks and dissections. In the sample soldered with VPS, the joints are more regular and have a significantly smoother surface. This better quality is probably caused by non-oxygen atmosphere of VPS (Fig. 2).
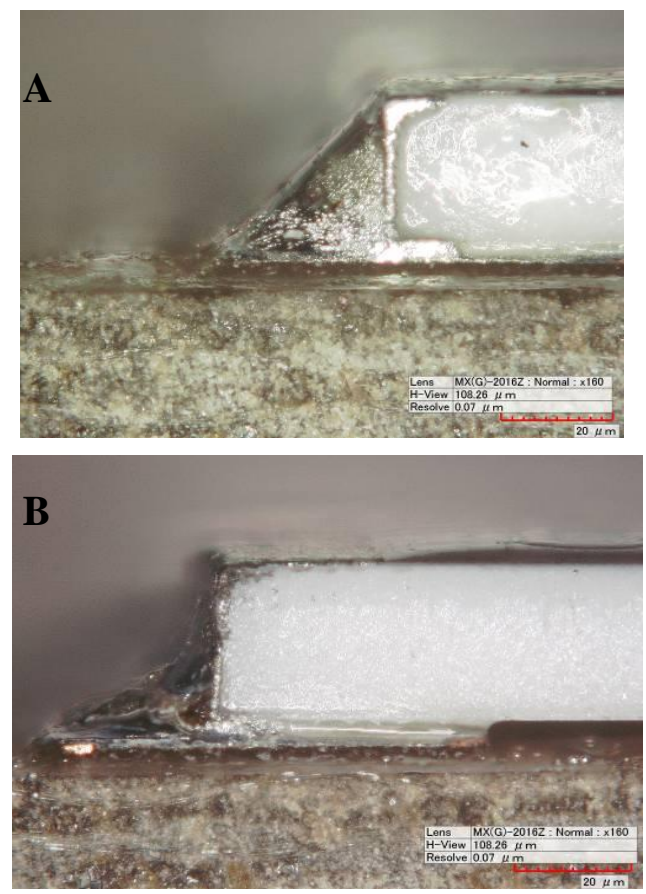

Fig. 2. Example optical images of $\mathrm{Sn} 96.5 \mathrm{Ag} 3 \mathrm{Cu} 0.5$ joints from samples with an Sn sublayer. A - VPS, B- reflow soldering.

\section{B. Mechanical Strength Investigation}

Mechanical strength is the most important indicator of joint performance. It determines whether the soldered element is attached strongly enough to the substrate. It can also give information on whether the soldering process was such that the junction was based on diffusion and not simply on adhesion. A high value of shear strength indicates a the thicker layer of intermetallic compounds which guarantee the durability of the connection. However too thick a layer of IMCs will lead to an increase in compressive stress and can also deteriorate joint quality.

To check the mechanical strength of the joints, the test samples were additionally subjected to 1500 temperature shocks within a cyclic temperature range of $-45^{\circ} \mathrm{C}$ to $\pm 85^{\circ} \mathrm{C}$, with each cycle lasting $20 \mathrm{~min}$. The transition time of the lift between hot and cold zones was only $5 \mathrm{~s}$ [Vötsch Chamber].

The results presented in Fig. 3 show a statistically important difference in the strength of joint connections dependent on sample type and also soldering technology.

As shown in Fig. 3, the results for the two soldering 
methods are at a high level (around 20-40 N). The relative success of the reflow method can be clue to the creation of intermetallic compounds (IMCs) with greater thickness, which brings a higher level of mechanical strength. The mechanical strength of a joint soldered with VPS is considerably dependent on the alloy used. As can be seen, statistically $\mathrm{Sn} 96.5 \mathrm{Ag} 3 \mathrm{Cu} 0.5$ produces significantly better results and better joint quality.

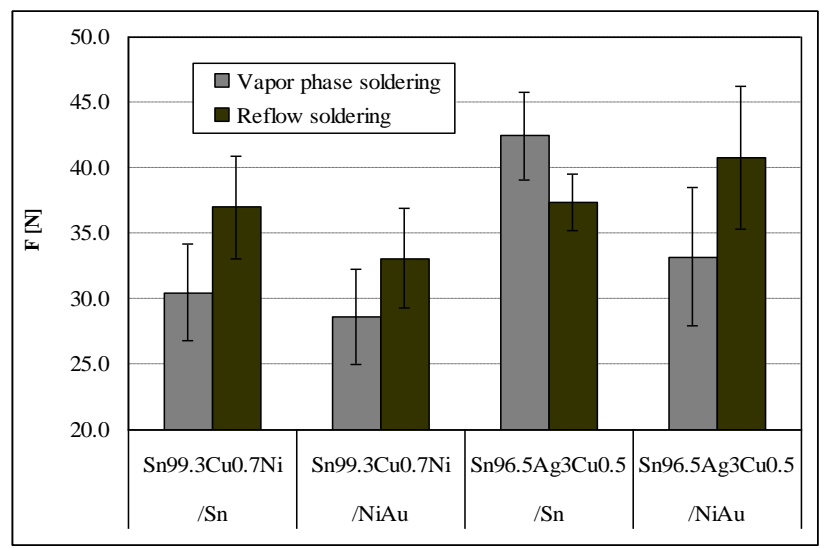

Fig. 3. Differences in strength force $[\mathrm{N}]$ dependent on sample type and soldering technology (reflow and VPS).

\section{Radioscopic Analysis}

Computed Tomography (CT) is a technique for producing 2-D and 3-D cross-sectional images of an object from flat $\mathrm{X}$-ray images.

This imaging technology utilizes an X-ray signal registered by a digital detector to nondestructively produce tomograms, and, as a result 'slices' of specific areas of the sample (free spaces can be separated from uniform material because of differential waves refraction). Analysis of the images was done by the 3D application MyVGL. 3D tomograms of solder joints were sliced in $\mathrm{Y}$ axes (with a distance of $0.024 \mathrm{~mm}$ ) to get cross-sectional images of the soldered material (Fig. 4).

Cross sections were taken in similar distances from the edges in both samples.

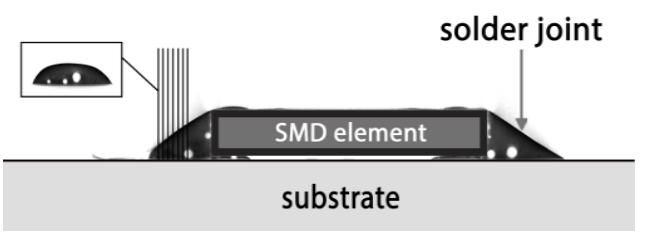

Fig. 4. Schematic slices in Y-axes tomogram of solder joint. Distance between cross sections: $0.024 \mathrm{~mm}$ (10 voxels in Nanotom specification).

Voids (cavities formed in solder joints) are common occurrence in SMT and are mainly caused by the amount of outgassing flux getting entrapped in the solder joint during the reflow process [9]. There are several sources of outgassing substance which may create voids. Generally it is produced by the evaporation of solvents and rheological additives during the reflow process. However it may also come straight from the metallization of the substrate, component or solder powder surface during the fluxing reaction [10]. This is dependent on the chemical composition of the paste and the medium. The main causes of void formation are inappropriate solder material and the nature of the soldering process, but the location and size of solder joints is also not irrelevant. Since gas bubbles have a lower density than molten solder paste, voids rise to the top of joints.

As can be seen in Fig. 5 void formation is very common in SMD soldering. Those parts of a joint hidden under SMD elements have less chance to release outgassing flux during the process. That is why in the total amount of voids is higher in such areas.

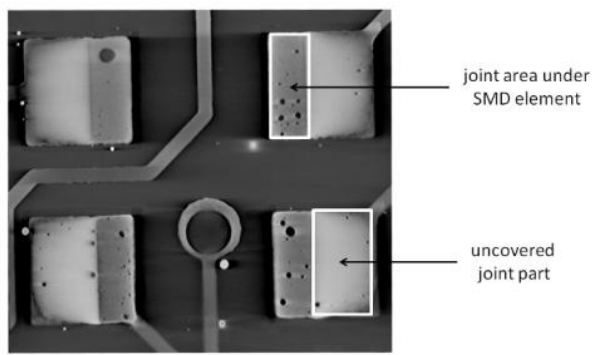

Fig. 5. X-Ray cross-section showing the arrangement of voids in solder joints (reflow process, paste $\mathrm{Sn} 96.5 \mathrm{Ag} 3 \mathrm{Cu} 0.5$ ).

The images presented in Fig. 6 show the amount and areas of internal voids in the cross-sections of samples produced by VPS and reflow soldering techniques. As can be noticed, both reflow soldering and VPS involve the occurrence of voids. The number of voids is comparable in both cases but in a sample soldered with the vacuum option, this amount is visibly lower than with traditional VPS. However, the diameters of voids in the reflow soldered joint are bigger, than in the case of VPS. The most important fact is that voids are especially visible at the interface of two layers, i.e.; the $\mathrm{Cu}$ and solder layers (pictures $\mathrm{A} 1, \mathrm{~B} 1$ and $\mathrm{C} 1$ show the arrangement of voids in an area of 45 microns from the edge of SMD).

VPS with the innovative vacuum system allows gasses to be sucked from joints during the soldering process (voids and enclosures can be eliminated almost completely). That is why samples made with the vacuum option have a significantly lower level of voids. However, the problem of releasing gas from parts hidden deep in the joint or even under the SMD element still exists. For better results, a good choice of medium fluid and solder alloys, effectively working in tandem with a vacuum and SMD materials are advised.

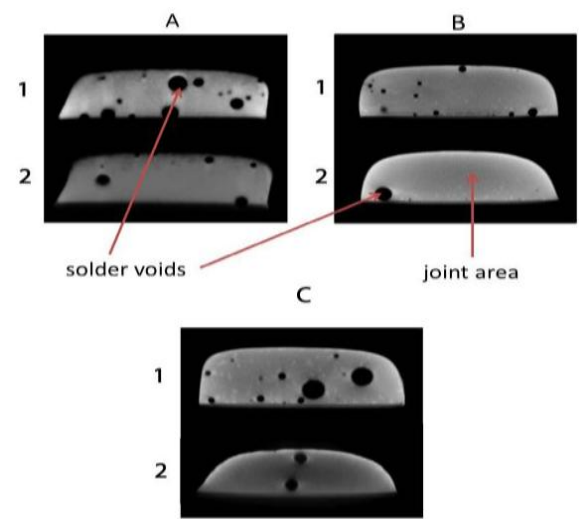

Fig. 6. Cross-section image of $\mathrm{Sn} 96.5 \mathrm{Ag} 3 \mathrm{Cu} 0.5$ joints soldered with: A reflow soldering, B - VPS with working vacuum, C - VPS without vacuum taken from an X-Ray tomogram. Black areas correspond with free spaces in material (voids). 


\section{CONCLUSIONS}

The reliability of an electronic device may depend on all soldered connections. It is important to select an appropriate method and to optimize the soldering process parameters. Vapor phase soldering has many advantages such as a lower degree of joint surface oxidation, the ability to achieve a good continuity of solder joint and a reduction of voids the formation of voids.

The results of the experiments involving VPS and reflow soldering techniques lead to the following conclusions:

1) Joints made with the VPS technique are more regular and have a significantly smoother surface.

2) The mechanical strength of a joint soldered with VPS is at a level comparable to joints made with a reflow technique.

3) The VPS technique influences the formation of voids. An especially positive influence is visible when the vacuum option is applied.

The above studies also suggest that the choice of solder alloy used for vapor phase soldering may be a key factor in the quality of the solder junction.

\section{REFERENCES}

[1] A. Rahn, The basics of soldering, John Wiley\&Sons INC., New York, 1993, pp. 2

[2] Y. Y. Wei and J. G. Duh, "Effects of thermal ageing on (Sn-Ag, Sn-Ag-Zn)/PtAg, Cu/A12O3 solder joints," Journal of Materials Science: Materials in Electronics, vol. 9, pp. 373-381, 1998.

[3] G. Gosh "Interfacial microstructure and the kinetics of interfacial reaction in diffusion couples between $\mathrm{Sn}-\mathrm{Pb}$ solder and $\mathrm{Cu} / \mathrm{Ni} / \mathrm{Pd}$ metallization," Acta Materialia, vol. 48, pp. 3719 - 3738, 2000.

[4] C. B. Lee, J. W. Yoon, S. J. Suth, S. B. Jung, C. W. Yang, C. C. Shur, and Y. E. Shin, "Intermatallic compound layer formation between Sn-3.5 mass \% Ag solder ball and $(\mathrm{Cu}$, immersion Au/electroless $\mathrm{Ni}-\mathrm{P} / \mathrm{Cu})$ substrate," Journal of Materials Science: Materials in Electronics, vol. 14, pp. 487 - 493, 2003.

[5] A. Skwarek, M. Pluska, J. Ratajczak, A. Czerwinski, K. Witek, and D. Szwagierczak, "Analysis of Tin Whisker Growth on Lead-Free Alloys with Ni Presence under Thermal Shock Stress," Materials Science and Engineering B, vol. 176, pp. 352-357, 2011.

[6] A. Skwarek, M. Sroda, M. Pluska, A. Czerwinski, J. Ratajczak, and K. Witek, "Occurence of Tin Pest on the Surface of Tin-Rich Lead-Free Alloys," Soldering and Surface Mount Technology, vol. 23 /3, pp. 184-190, 2011.
[7] I. Plotog, G. Varzaru, and P. Svasta, "VPS Technology and Applications," in Proc. $3^{\text {rd }}$ International Symposium on Electrical and Electronics Engineering, Galati, September 2010.

[8] X. Liu and G. Q. Lu, "Effects of solder joint shape and height on thermal fatigue lifetime," IEEE Transactions on Components and Packaging Technologies, vol. 26, no. 2, 2003.

[9] W. B. O'Hara and N. C. Lee, "Voiding in BGAs," Soldering and Surface Mount Technology, vol. 7, no. 3, pp. 44-48, 1995

[10] A. A. Primavera, R. Strum , S. Prasad, and K. Srihari, "Factors that affect void formation," in Proc. Surface Mount Technology Association, 1998.

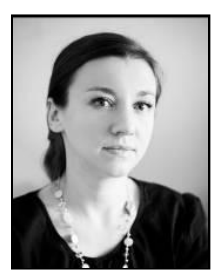

Agata Skwarek was born in Krakow, Poland in May 1979. In 2003 she graduated from the Faculty of Chemistry at the Jagiellonian University. In 2010, she defended her doctoral dissertation in technical science entitled "Analysis of tin whiskers and tin pest influencing the quality of tin-rich Pb-free solder joint" in Institute of Electron Technology, Poland. In 2012 she finished annual postgraduate „Manager of Research and Development" organized by the Polish Foundation of Opportunities Industrialization Centers "OIC Poland" and University of Economics and Innovation in Lublin, Poland.

From 2004, she is working in the Institute of Electron Technology in Krakow Division. In 2004-2011 she worked as an assistant and form 2011 as an assistant professor. From 2006 to 2013 she participated in several training courses in the field of electronics and project management. She has authored/co-authored over dozen articles published in international scientific journals, two patent applications and two patents. She also participated in the development of several models and prototypes. She participated as project executive in six research projects. Twice she was also project manager. Actually she acts in the field of microelectronics in particular on the reliability of solder joints in the test circuits.

Dr. Skwarek is a member of International Microelectronics and Packaging Society (IMAPS Poland Chapter). In 2007 she received the conference scholarship of the Foundation of Polish Science, for the presentation of the article entitled „Influence of Environmental Conditions on Pb-free Solder Joints Quality”. In 2008 she was awarded with „Highly Commended Award" by the Emerald publisher for the article "Fabrication and characterization of bulk and thick film perovskite NTC thermistors" (Emerald Literati Network 2008 Awards for Excellence). Also in 2008 she was awarded for taking second place at $32^{\text {nd }}$ International Microelectronics and Packaging IMAPS-CPMT Poland Conference for the poster entitled „Risk of Tin Whiskers Formation on the Surface of Tin-rich Alloys under Thermal Shock Stress".

In 2006 and 2013 she worked in the Organizing Committee of International Conference of IMAPS Poland Chapter 2006, Kraków. 